\title{
CONJECTURING THE MATHEMATICAL AXIOM THAT PROVIDES A UNIFIED THEORY OF RESONANCE AND DECAY AND CONNECTS IT TO CAUSAL TIME EVOLUTION
}

\author{
ARNO BOHM AND PETER BRYANT
}

Communicated by Piotr Kielanowski

\begin{abstract}
Combining the ideas of causality and the phenomenology of resonances and decaying states, we modify standard quantum theory by changing one of its axioms. The first step was taken decades ago when Dirac kets were given a mathematical meaning as functionals on a Schwartz space, which led to a Gel'fand triplet in which observables were represented by an algebra of continuous operators. The second step, motivated by the analytic continuation to complex energies for the S-matrix and the two Lippmann-Schwinger kets, distinguishes between prepared in-states-vectors and detected out-vectors. This leads to the pair of Gel'fand triplets realized by Hardy functions of the upper (for prepared states) and lower (for detected observables) complex energy plane. Replacing the Hilbert space axiom of quantum mechanics by the Hardy space axiom, one obtains a mathematically consistent theory that unifies resonance and decay phenomena and that has a causal, asymmetric time evolution.
\end{abstract}

\section{Introduction}

Time asymmetry is the most prevalent feature of our world. In physics it has not been ignored, but it has been neglected. In quantum physics, it has been unjustly neglected. One usually considers situations that are too idealized, and one investigates problems for which the directedness of time and for which irreversibility do not play a prominent role. An example is classical mechanics, where one deals mostly with conservative forces and treats friction as an addendum.

Quantum mechanics falls into roughly two categories.

I. Spectra and structure of micro-physical systems in stationary states. It uses the Hilbert space boundary condition for the dynamical equation (Schrödinger or Heisenberg). As a consequence, the dynamical evolution is described by the reversible unitary group evolution. 
II. Dynamically evolving states, resonance and decay phenomena.

The time symmetric unitary group does not adequately describe their dynamical evolution. Within the constraints of the Hilbert space axiom, "there does not exist. . . a rigorous theory to which" the Weisskopf-Wigner methods used for these phenomena "can be considered as approximations." [11]

\section{Standard Quantum Mechanics Theory is Time Symmetric}

In the early development of the foundations of quantum theory, the mathematics available and provided by von Neumann's Hilbert space axiom [19] led to a time symmetric evolution, $\phi(t)=U^{\dagger}(t) \phi(0)$, given by the unitary group, $U^{\dagger}(t)=$ $\mathrm{e}^{-\mathrm{i} H t / \hbar}$ for $-\infty<t<+\infty$.

In the foundations of quantum mechanics one distinguishes between states and observables [12]. States are described by density operators, $\rho$, or by vectors, $\phi$, for pure states. Observables are described by operators, $A\left(=A^{\dagger}\right), \Lambda$, or by vectors, $\psi$, if $\Lambda=|\psi\rangle\langle\psi|$. The state $\rho$ (for instance the in-state $\rho=\left|\phi^{+}\right\rangle\left\langle\phi^{+}\right|$of a scattering experiment) is prepared by a preparation apparatus (accelerator). The observable $A$ (for instance the out-observable $A=\left|\psi^{-}\right\rangle\left\langle\psi^{-}\right|$of a scattering experiment) is registered or detected by a registration apparatus (detector). The time evolution is given by the dynamical equations: the Schrödinger equation for the states $\phi^{+}$

$$
\mathrm{i} \hbar \frac{\mathrm{d}}{\mathrm{d} t} \phi^{+}(t)=H \phi^{+}(t)
$$

or the Heisenberg equation for the observables $\psi^{-}$or $\Lambda$

$$
\mathrm{i} \hbar \frac{\mathrm{d}}{\mathrm{d} t} \psi^{-}(t)=-H \psi^{-}(t), \quad \mathrm{i} \hbar \frac{\mathrm{d}}{\mathrm{d} t} \Lambda(t)=[\Lambda(t), H] .
$$

To solve these dynamical differential equations, one needs boundary conditions, for which von Neumann provided the Hilbert space axiom

set of states $\left\{\phi^{+}\right\}=$set of observables $\left\{\psi^{-}\right\}=\mathcal{H}=$ Hilbert space.

Solving (2.1) and (2.2) under the boundary conditions (2.3), one obtains as the general solutions (Stone-von Neumann theorem) [17]

$$
\phi^{+}(t)=\mathrm{e}^{-\mathrm{i} H t / \hbar} \phi^{+}(0), \quad-\infty<t<\infty
$$

for states $\left|\phi^{+}(t)\right\rangle\left\langle\phi^{+}(t)\right|$, and

$$
\psi^{-}(t)=\mathrm{e}^{\mathrm{i} H t / \hbar} \psi^{-}(0), \quad \Lambda(t)=\mathrm{e}^{\mathrm{i} h t / \hbar} \Lambda \mathrm{e}^{-\mathrm{i} H t / \hbar}, \quad-\infty<t<\infty
$$


for observables $\left|\psi^{-}(t)\right\rangle\left\langle\psi^{-}(t)\right|$ or $\Lambda(t)$. This is the time symmetric evolution given by the group

$$
U^{\dagger}(t)=\mathrm{e}^{-\mathrm{i} H^{\dagger} t / \hbar}, \quad-\infty<t<\infty
$$

with the group product

$$
U^{\dagger}\left(t_{1}\right) U^{\dagger}\left(t_{2}\right)=U^{\dagger}\left(t_{1}+t_{2}\right)
$$

For every evolution $U^{\dagger}(t)$ there exists the inverse

$$
U^{\dagger-1}(t)=U^{\dagger}(-t)
$$

so that the evolution is reversible for the state (2.4) or for the observable (2.5).

In quantum mechanics, the experimentally observed quantities are the probabilities to measure the observable $\Lambda$ in the state $\rho: \mathcal{P}_{\rho}(\Lambda(t))$. They are calculated in theory as the Born probabilities [3]

$$
\mathcal{P}_{\rho(t)}\left(\Lambda_{0}\right) \equiv \operatorname{Tr}\left(\Lambda_{0} \rho(t)\right)
$$

in the Schrödinger picture, or for the special case $\Lambda_{0}=\left|\psi_{0}^{-}\right\rangle\left\langle\psi_{0}^{-}\right|$and $\rho(t)=$ $\left|\phi^{+}(t)\right\rangle\left\langle\phi^{+}(t)\right|$

$$
\mathcal{P}_{\phi^{+}(t)}\left(\psi_{0}^{-}\right)=\left|\left\langle\psi_{0}^{-} \mid \phi^{+}(t)\right\rangle\right|^{2} .
$$

In the Heisenberg picture the Born probabilities are

$$
\mathcal{P}_{\rho_{0}}(\Lambda(t)) \equiv \operatorname{Tr}\left(\Lambda(t) \rho_{0}\right)
$$

or for the special case $\Lambda(t)=\left|\psi^{-}(t)\right\rangle\left\langle\psi^{-}(t)\right|$ and $\rho_{0}=\left|\phi_{0}^{+}\right\rangle\left\langle\phi_{0}^{+}\right|$

$$
\mathcal{P}_{\phi_{0}^{+}}\left(\psi^{-}(t)\right)=\left|\left\langle\psi^{-}(t) \mid \phi_{0}^{+}\right\rangle\right|^{2} .
$$

Here $\phi_{0}^{+}\left(\rho_{0}\right)$ is the state prepared at a particular time $t_{0}(=0)$. The Born probabilities are measured experimentally as ratios of large (sometimes not so large) numbers of detector counts.

$$
\mathcal{P}_{\rho_{0}}(\Lambda(t))=\mathcal{P}_{\rho(t)}\left(\Lambda_{0}\right) \approx \frac{N(t)}{N_{\text {total }}} .
$$

The time evolution of the quantum mechanical probabilities under the standard Hilbert space axiom (2.3) is therefore given by

$$
\mathcal{P}_{\rho(t)}\left(\Lambda_{0}\right)=\operatorname{Tr}\left(U^{\dagger}(t) \rho(0) U(t) \Lambda_{0}\right)=\operatorname{Tr}\left(\rho(0) U(t) \Lambda_{0} U^{\dagger}(t)\right)
$$


or for the special case

$$
\begin{aligned}
\mathcal{P}_{\phi^{+}(t)}\left(\psi_{0}^{-}\right) & =\left|\left\langle\psi_{0}^{-} \mid \phi^{+}(t)\right\rangle\right|^{2}=\left|\left\langle\psi_{0}^{-} \mid \mathrm{e}^{-\mathrm{i} H t / \hbar} \phi_{0}^{+}\right\rangle\right|^{2} \\
& =\left|\left\langle\mathrm{e}^{\mathrm{i} H t / \hbar} \psi_{0}^{-} \mid \phi_{0}^{+}\right\rangle\right|^{2}=\left|\left\langle\psi^{-}(t) \mid \phi_{0}^{+}\right\rangle\right|^{2} \\
& =\mathcal{P}_{\phi_{0}^{+}}\left(\psi^{-}(t)\right), \quad-\infty<t<+\infty
\end{aligned}
$$

where $\phi_{0}^{+}$is the state at a particular time, $t_{0}=0$, at which the state is prepared. If the Hilbert space axiom, and consequently the unitary group time evolution, holds, then the Born probabilities to detect the observable $|\psi\rangle\langle\psi|$ are predicted for all values of time: $-\infty<t<\infty$. If $t_{0}=0$ is the time at which the state $\phi_{0}^{+}$ is prepared, then according to (2.4) the Born probability as a function of time can evolve forward with the state, evolving as $\phi_{0}^{+} \longrightarrow U^{\dagger}(t) \phi_{0}^{+}=\phi^{+}(t), t>0$. It can also evolve backward with the state, $\phi_{0}^{+} \longrightarrow\left(U^{\dagger}(t)\right)^{-1} \phi_{0}^{+}=U^{\dagger}(-t) \phi_{0}^{+}=$ $\phi^{+}(-t)$. A reversible evolution for probabilities, whether they are classical or quantal probabilities, seems odd. But this is what the Hilbert space axiom (2.3) of standard quantum mechanics predicts.

As long as time evolution is not an issue, the standard axioms of quantum mechanics, of which there are about six [3], work fine, especially if one also uses Dirac kets and the continuous basis vector expansion.

This is Category I quantum mechanics, which describes the structures and spectra of micro-physical systems in stationary states

$$
\rho(t) \equiv U^{\dagger}(t) \rho U(t)=\rho .
$$

This applies to the ground states of atoms, molecules, nuclei, and hadrons, but it is also used for singly excited states when they are treated like stable states. One calculates the energy spectra, the charge distribution, the multipole moments, etc., of atoms and molecules and infers from this their structure. The non-zero electric and magnetic moments imply transition and decay rates, $\mathcal{R}$, and finite lifetime, but one does not study the time evolution of these transitions. Instead one gives the initial transition probability per unit time (the decay rate at a time $t=0$ ) and uses it as a measure of the intensity for the transition. Transition rates are calculated at a time $t=0$. Scattering cross sections are expressed in terms of initial transition rates (Born probabilities per unit time at a mysterious time $t=0$ ) though the scattering process may continue for a long time as measured by the clock of the experimenter. For Category I problems at times $t=0$, the standard axioms of quantum mechanics suffice because, for them, time evolution is not an issue. One uses mostly the algebraic properties of the Hilbert space and not its topological properties (definition of convergence). 
Category II quantum mechanics deals with scattering and decay phenomena, continuous energy spectra for resonance scattering, and non-trivial time evolution for decay. If one wants to use continuous energy eigenstates, then this requires already more than the conventional axioms of quantum mechanics are able to accommodate. This has been handled by use of the Dirac kets $|E\rangle$, which - if they have been defined at all [18] - are defined as functionals on the Schwartz space. With this definition, the energy wave functions $\psi(E)=\langle E \mid \psi\rangle$ do not make up the whole Hilbert space of (Lebesgue) square integrable functions, but only the subspace of infinitely differentiable, rapidly decreasing functions, i.e., the Schwartz space functions.

The introduction of Dirac kets augments the conventional axiomatic framework of quantum mechanics based on the Hilbert space axiom (2.3) and leads to the Gel'fand triplet $\Phi \subset \mathcal{H} \subset \Phi^{\times}$, where $\Phi$ is the abstract Schwartz space. The Gel'fand triplet based on the Schwartz space, however, is not sufficient to obtain a theory that includes scattering and decay. The reason is that the dynamical (Schrödinger or Heisenberg) equations, when defined as differential equations in the Schwartz space, also integrate to a continuous group evolution much like (2.4) and (2.6).

In contrast, resonances and decaying states have been intuitively associated with an asymmetric, "irreversible" time evolution [13]. Thus, they require a time asymmetric theory, and in the absence of such a mathematical theory, their description can only be approximate [11] and must contain contradictions.

\section{Resonances and Decay}

Experiments show that all spontaneously decaying quantum systems obey the exponential decay law $\mathcal{P}(t) \sim \mathrm{e}^{-t / \tau}$. This exponential law can be considered as one of the best established laws of nature [15]. Therefore one expects that there are plenty of state vectors, $\phi^{G}(t)$, for which one can calculate the Born probabilities for any observable $|\psi\rangle\langle\psi|$

$$
\mathcal{P}_{\phi^{G}(t)}(\psi)=\left|\left\langle\psi \mid \phi^{G}(t)\right\rangle\right|^{2}
$$

and the decay rate

$$
\mathcal{R}_{\phi^{G}(t)}(\psi)=\frac{\mathrm{d} \mathcal{P}_{\phi^{G}(t)}(\psi)}{\mathrm{d} t}=\frac{\mathrm{d}}{\mathrm{d} t}\left|\left\langle\psi \mid \phi^{G}(t)\right\rangle\right|^{2} .
$$

A decaying state, $\phi^{G}$, is characterized by its energy $E_{D}$ and the value of its life- 
time $\tau$. Its lifetime is measured by a fit of the decay rate to the exponential law

$$
\mathcal{R}(t) \approx \frac{\Delta N\left(t_{i}\right)}{N_{\text {total }} \Delta t_{i}} \propto \mathrm{e}^{-\frac{t}{\tau}}
$$

A decaying state $\phi^{G}$ is characterized by the values $\left(E_{D}, \tau\right)$ and the other quantum numbers, angular momentum $j$, etc., as well: $\left(E_{D}, \tau\right), j, j_{3}, \eta$. There are many examples for a wide range of values of $\left(E_{D}, \tau\right)$. If, however, one accepts the Hilbert space axiom and is thereby restricted to states represented by vectors in the Hilbert space, then there is no state having exponential decay. This is the consequence of a mathematical theorem [10].

For the theoretical description of resonance scattering and decay one uses continuous energy eigenvalues, $0 \leq E<\infty$, and even eigenvectors of the Hamiltonian, $H$, with complex energy eigenvalues. Such tools are not well defined for quantum mechanical entities represented by Hilbert space vectors.

A resonance is characterized by its energy $E_{R}$ and a definite value of its Lorentzian width $\Gamma$ in the scattering amplitude. The scattering amplitude with angular momentum $j$ is

$$
a_{j}(E)=\frac{r_{\eta}}{E-\left(E_{R}-\mathrm{i} \frac{\Gamma}{2}\right)}+B(E)=a_{j}^{B W}(E)+B(E) .
$$

The slowly varying function $B(E)$ is the background, which changes from process to process. The function $a_{j}^{B W}(E)$ is a Breit-Wigner amplitude of the resonance, and it is the same for every process in which this resonance appears. The width $\Gamma$ and the resonance energy $E_{R}$ are measured by a fit of the cross section data to

$$
\sigma_{j}(E) \sim\left|a_{j}(E)\right|^{2} .
$$

Many people think that resonances and decaying states are the same physical entities

$$
\text { resonances }=\text { decaying states }
$$

and that, at least for non relativistic quantum theory, the width and the inverse lifetime are the same, except for the factor of $\hbar$

$$
\frac{\hbar}{\Gamma}=\tau \equiv \frac{1}{\mathcal{R}} .
$$

The $\equiv$ in (3.6) is an identity if the exponential law holds.

Within the framework of standard quantum theory under the Hilbert space boundary conditions (2.3) for the dynamical equations (2.1) and (2.2), one cannot obtain a theory that unifies resonance scattering and decay and that predicts (3.6). 
The closest one can get to the relation (3.6) is the Weisskopf-Wigner (WW) approximation [20]. Using Weisskopf-Wigner methods, the probability of measuring a prepared resonance state $\phi(t)$ with Breit-Wigner width $\Gamma$ is [21]

$$
\mathcal{P}_{\phi(t)}(\psi) \sim \mathrm{e}^{-\Gamma t / \hbar}+\frac{\Gamma}{E_{R}} \times(\text { additional terms }(t)) .
$$

Thus one may be inclined to give the impression that, in the limit $\frac{\Gamma}{E_{R}} \rightarrow 0$, the exponential law for the probability rate (3.2) follows and then to compare this with (3.3) to relate the lifetime, $\tau$, to the width, $\Gamma$. However, “... there does not exist. . . a rigorous theory to which these various [WW] methods can be considered as approximations" [11].

To obtain a theory that unifies Breit-Wigner resonance scattering (3.4) with exponential decay (3.3), one needs a state vector $\phi^{G}$ with the energy distribution $a_{j}^{B W}(E)$ of (3.4) and with exactly exponential time evolution

$$
\operatorname{Tr}\left(|\psi\rangle\left\langle\psi \mid \phi^{G}(t)\right\rangle\left\langle\phi^{G}(t)\right|\right)=\mathcal{P}_{\phi^{G}(t)}(\psi) \propto \mathrm{e}^{\frac{-\Gamma t}{\hbar}}
$$

for all decay products $|\psi\rangle\langle\psi|$. Such a vector does not exist in the Hilbert space.

The simplest way to derive an exactly exponential decay probability is to postulate a state vector $\phi^{G}$, which has the properties

$$
H \phi^{G}=\left(E_{R}-\mathrm{i} \frac{\hbar \mathcal{R}}{2}\right) \phi^{G} \text { and } \phi^{G}(t)=\mathrm{e}^{\frac{-\mathrm{i} H t}{\hbar}} \phi^{G} .
$$

Then the decay probability of $\phi^{G}(t)$ into any observable $|\psi\rangle\langle\psi|$ is

$$
\begin{aligned}
\mathcal{P}_{\phi^{G}(t)} & =\left|\left\langle\psi \mid \phi^{G}(t)\right\rangle\right|^{2}=\left|\left\langle\psi\left|\mathrm{e}^{\frac{-\mathrm{i} H t}{\hbar}}\right| \phi^{G}\right\rangle\right|^{2} \\
& =\left|\left\langle\psi \mid \phi^{G}\right\rangle \mathrm{e}^{-\mathrm{i}\left(E_{R}-\mathrm{i} \hbar \mathcal{R} / 2\right) t / \hbar}\right|^{2}=\left|\left\langle\psi \mid \phi^{G}\right\rangle\right|^{2} \mathrm{e}^{-\mathcal{R} t} .
\end{aligned}
$$

The vector $\phi^{G}$ is called a Gamow vector [7], and it is not found in standard, Hilbert space quantum mechanics because

1. It would have to be a vector with a complex eigenvalue for a selfadjoint Hamiltonian $H$ and thus could at best be a generalized vector like the Dirac kets: $\phi^{G} \sim\left|E_{R}-\mathrm{i} \frac{\hbar \mathcal{R}}{2}\right\rangle$.

2. As a consequence of (2.4), the time extends for all solutions of the Schrödinger equation over $-\infty<t<\infty$. Thus the exponential in (3.10) would diverge and lead to the "exponential catastrophe." 
To obtain a Gamow vector, one would require a theory that admits complex energy eigenvalues $z_{R}=\left(E_{R}-\mathrm{i} \frac{\hbar \mathcal{R}}{2}\right)$, which fulfill the relation $\hbar R=\Gamma$ where $\Gamma$ is measured by (3.5), and that has asymmetric time evolution, $t_{0} \leq t<\infty$. That this can indeed be accomplished by a consistent mathematical theory will be explained in Section 5.

\section{Time Asymmetry}

Because time asymmetry emerged as a consequence of the mathematical conditions needed to construct a theory that unifies resonance scattering and decay [1], we want to discuss in this section the evidence for time asymmetry in quantum physics. We shall see that instead of starting from the idea of a unified quantum theory of resonance and decay phenomena and arriving at time asymmetry, one could as well have started from the idea of time asymmetric quantum theory and conjectured a consistent theory that unifies resonances and decay phenomena.

Time asymmetry and "Arrows of Time" are well known from classical physics. The best known arrow of time is the Thermodynamic Arrow of Time, by which the entropy, $S$, in an isolated (classical) system increases, $\frac{\mathrm{d} S}{\mathrm{~d} t}>0$, until it reaches equilibrium, $\frac{\mathrm{d} S}{\mathrm{~d} t}=0$. Peierls [16] explains this irreversibility as a consequence of time asymmetric boundary conditions implied by Boltzmann's Stosszahl Ansatz for the time symmetric equations of classical mechanics.

The Radiation Arrow of Time is a well known consequence of a time asymmetric boundary condition. The Maxwell's equations, like all local laws of physics, are symmetric in time. But boundary conditions (the Sommerfeld radiation conditions) exclude the strictly incoming fields, $A_{\text {in }}^{\mu}=0$, and select only the retarded fields of the other particles in the region

$$
A^{\mu}(x)=A_{\text {ret }}^{\mu}(x)+A_{\text {in }}^{\mu}(x)=A_{\text {ret }}^{\mu}(x) .
$$

Radiation must be emitted by a source before it can be detected by a receiver.

There does not seem to be an empirical reason to forbid time asymmetric boundary conditions for the dynamical equations (Schrödinger or Heisenberg equations) of quantum mechanics. Still, the widespread opinion is that a theory of irreversible, time asymmetric evolution for isolated quantum systems is an impossibility. Several physicists, however, have considered time asymmetry or irreversibility of the time evolution for quantum systems.

Peierls and his school [9] emphasized the importance of the initial conditions and boundary conditions. Instead of the axiom (2.3), they chose purely outgoing 
boundary conditions for the solutions of the Schrödinger equation. In their view, irreversibility is a consequence of the choice of boundary condition. They may not have realized that time asymmetry, $t_{0} \leq t<\infty$, together with the dynamical equations can lead to a semigroup time evolution.

In scattering theory (not quite Hilbert space quantum mechanics), one often uses the retarded propagator, or Green's function [14]

$$
\mathcal{G}^{+}(t)= \begin{cases}\mathrm{e}^{-\mathrm{i} H t} & \text { for } t \geq 0 \\ 0 & \text { for } t<0\end{cases}
$$

which describes the time asymmetric evolution of the prepared in-state vector,

$$
\phi(t)=\mathcal{G}^{+}\left(t-t_{0}\right) \phi\left(t_{0}\right), \quad t \geq t_{0} .
$$

In Hilbert space, such semigroup solutions of the Schrödinger equation do not exist.

Lee $[13 \mathrm{c}$ ] called this time asymmetry the "impossibility of constructing timereversed quantum solutions for a micro-physical system" (decaying systems). His arguments immediately extend to scattering experiments:

It is easy to prepare two uncorrelated, incoming beams that scatter into strongly correlated, outgoing spherical waves, as done in a typical scattering experiment. It is experimentally hopeless to prepare a state consisting of two strongly correlated, spherical waves (with fixed relative phase) in such a way that after the scattering, two uncorrelated plane waves emerge. The latter would be the time reverse of the setup for a physical scattering experiment.

Ludwig [12] noticed that, in experiments with quantum systems, one could not time-translate the trigger of a detector to a time before the preparation apparatus (accelerator) had been turned on. In other words, the preparation of a state at $t_{0}=0$ must precede the registration of an observable in that state at a later time $t>t_{0}$. Therefore, time translations form a semigroup. This semigroup for time translation of the apparatuses ought to be transcribed into a semigroup evolution of the state (defined by the preparation apparatus) relative to the observable (defined by the registration apparatus). Knowing that under the Hilbert space axiom (2.3) the time evolution is represented by the unitary group, however, Ludwig extrapolated this semigroup to all times $-\infty<t<\infty$, hence the group (2.6)-(2.8). Gell-Mann and Hartle [8] introduced an arrow of time in the quantum mechanics of cosmology. To avoid inconsistencies for the probabilities of histories, they required a time ordering for the projection operators in a history and in the initial states. This time ordering "may not be attributed to the thermodynamic arrow of 
an external measuring apparatus or larger universe," but it "would be a fundamental quantum mechanical distinction between the past and the future."

They introduced the semigroup operator by decree (citing Feynman): The state, $\rho$, of our universe, considered as a quantum physical system, evolves

$$
\rho(t)=\mathrm{e}^{-\mathrm{i} H\left(t-t_{0}\right)} \rho\left(t_{0}\right) \mathrm{e}^{\mathrm{i} H\left(t-t_{0}\right)}, \quad \text { only for } t \geq t_{0} \equiv t_{\text {big bang }} .
$$

Quantum mechanical probability for the observable $P_{\alpha_{1}}=\left|\psi_{\alpha_{1}}\right\rangle\left\langle\psi_{\alpha_{1}}\right|$

$$
\begin{aligned}
\mathcal{P}_{\rho}\left(P_{\alpha_{1}}\left(t_{1}\right)\right) & =\operatorname{Tr}\left(P_{\alpha_{1}}\left(t_{1}\right) \rho\left(t_{0}\right)\right)=\operatorname{Tr}\left(\mathrm{e}^{\mathrm{i} H t_{1}} P_{\alpha_{1}} \mathrm{e}^{-\mathrm{i} H t_{1}} \rho\left(t_{0}\right)\right) \\
& =\operatorname{Tr}\left(P_{\alpha_{1}} \rho\left(t_{1}\right)\right)
\end{aligned}
$$

can exist for $t_{1} \geq t_{\text {big bang. The time evolution operator was written in the form }}$

$$
U(t)=\left.\mathrm{e}^{\mathrm{i} H t}\right|_{t \in \mathbb{R}_{+}}, \quad \text { for } t_{\text {big bang }} \leq t<\infty
$$

with $t_{\text {big bang }}=0$ chosen as the beginning of time. The experimental evidence for this time asymmetry is the Big Bang, at which time our universe was supposedly a quantum system. The mathematical meaning of the operators $U$ and $H$ in (4.5) needs to be defined.

The idea that time asymmetry requires different boundary conditions for the set of vectors $\phi$ used for states and the set of vectors $\psi$ used for observables, thereby mathematically distinguishing states from observables, is contained in the famous article of Feynman [5]: "We choose a particular time $t=t_{0}$ and divide the region ...into

a) a region $R^{\prime} \ldots$ such that $t^{\prime}<t_{0}$, and...

b) a region $R^{\prime \prime} \ldots$ such that $t^{\prime \prime}>t_{0}$."

"The state at $t^{\prime}$ is defined completely by the preparation [prepared $i n$-state $\phi^{+}$] . . Likewise the state characteristic of the experiment [registered observable $\psi^{-}$] can be defined by $\psi\left(t^{\prime \prime}\right) \ldots$ at time $t^{\prime \prime}>t_{0}$." "[T] in state $\phi\left(t^{\prime}\right)$ at time $t^{\prime}$ will be found after $t^{\prime \prime}$ to be in a state $\psi\left(t^{\prime \prime}\right)$ is the square of the transition amplitude" $\left(\psi\left(t^{\prime \prime}\right), \phi\right)$. (The symbols here are chosen to agree with those used on the proceeding pages; the words are from [5]. Feynman clearly distinguishes between prepared states and "states characteristic of the experiment." In place of the latter we use the word "observables.")

The square of the transition amplitudes are the Born probabilities, (2.9)-(2.12), and, according to Feynman's quote above, these Born probabilities can be defined 
for $t^{\prime \prime}>t_{0}$ only. Thus, Born probabilities do not exist for $t<t_{0}$, in contradiction with (2.15).

The probability to measure the observable $\psi\left(t^{\prime \prime}\right)$ in the state $\phi$ can be observed only at $t^{\prime \prime}>t_{0}$, because

A state must be prepared before an observable can be measured in it.

This statement, which is an expression of causality, is the quantum mechanical arrow of time. It states that if $t_{0}(=0)$ is the time at which the state $\rho$ has been prepared, then the probability $\mathcal{P}_{\rho}(\Lambda(t))$ is measured as the ratio of detector counts $\frac{N(t)}{N_{\text {total }}}$

$$
\mathcal{P}_{\rho}(\Lambda(t)) \approx \frac{N(t)}{N_{\text {total }}}, \quad \text { for } t \geq t_{0} \text { only. }
$$

At times $t<t_{0}$ no counts can be registered: $N(t)=0$ for $t<t_{0}$. Nothing can be observed in a state $\rho=|\phi\rangle\langle\phi|$ before that state has been prepared. Therefore

$$
\begin{aligned}
\mathcal{P}_{\rho}(\Lambda(t)) & =\operatorname{Tr}(|\psi(t)\rangle\langle\psi(t) \mid \phi\rangle\langle\phi|)=|\langle\psi(t) \mid \phi\rangle|^{2} \\
& =\operatorname{Tr}(|U(t) \psi\rangle\langle U(t) \psi \mid \phi\rangle\langle\phi|) \\
& =|\langle U(t) \psi \mid \phi\rangle|^{2}=\left|\left\langle\mathrm{e}^{\mathrm{i} H t} \psi \mid \phi\right\rangle\right|^{2} \\
& =\left|\left\langle\psi \mid U^{\times}(t) \phi\right\rangle\right|^{2}=\left|\left\langle\psi \mid \mathrm{e}^{-\mathrm{i} H^{\times}} \phi\right\rangle\right|^{2}, \quad t \geq t_{0}=0 .
\end{aligned}
$$

Thus the evolution operator, $U^{\times}(t)=\mathrm{e}^{-\mathrm{i} H^{\times} t / \hbar}$ for a state $\rho$ or $|\phi\rangle\langle\phi|$, and its adjoint (with $H \subset \bar{H}=H^{\dagger} \subset H^{\times}$), the evolution operator $U(t)=\mathrm{e}^{\mathrm{i} H t / \hbar}$ for observables $\Lambda$ or $|\psi\rangle\langle\psi|$, do not have any physical counterpart for $t<t_{0}=0$. The operator $A^{\times}$is the conjugate of an operator $A$. It is an extension of the Hilbert space adjoint operator $A^{\dagger}$ of $\bar{A}$, the closure of $A$. This will be discussed in Section 5.

As a consequence of the quantum mechanical arrow of time, (4.6)-(4.8), we conclude:

In the Schrödinger picture, the time evolved state

$$
\phi(t)=\mathrm{e}^{-\mathrm{i} H^{\times} t / \hbar} \phi \quad \text { is physically defined only for } t>t_{0}=0 .
$$

In the Heisenberg picture, the time translated observable

$$
\psi(t)=\mathrm{e}^{\mathrm{i} H t / \hbar} \psi \quad \text { is physically defined only for } t>t_{0}=0 .
$$

The time evolution is asymmetric, $0 \leq t<\infty$, and is given by the semigroup

$$
U^{\times}(t)=\mathrm{e}^{-\mathrm{i} H^{\times} t / \hbar} \quad \text { with } 0 \leq t<\infty \text {, for the states } \phi \text { or } \rho
$$


and by the semigroup

$$
U(t)=\mathrm{e}^{\mathrm{i} H t / \hbar} \quad \text { with } 0 \leq t<\infty \text {, for the observables } \psi, \Lambda \text {, or } A \text {. }
$$

Being a semigroup means that the product of two elements exists

$$
U^{\times}\left(t_{1}\right) U^{\times}\left(t_{2}\right)=U^{\times}\left(t_{1}+t_{2}\right)
$$

but the inverse, $U^{\times-1}(t)$, of an element, $U^{\times}(t), t>0$, does not exist.

The vectors $\phi(t)$ and $\psi(t)$ cannot be in one-to-one correspondence with the Hilbert space vectors of (2.4) and (2.5). And $U(t), U^{\times}(t), H$, and $H^{\times}$are not the selfadjoint $H=H^{\dagger}$ and unitary operators $U$ and $U^{\dagger}=U^{-1}$ of Section 2. They are new operators in new spaces that need to be mathematically defined. The similarity in the notation indicates that there is a relation between these operators and spaces and the operators and vectors in Hilbert space. But these new entities must first be mathematically defined before they can be employed to represent causal semigroups.

\section{A Time Asymmetric Theory Relating Resonances and Decaying States}

In Section 3 and Section 4 we have discussed the shortcomings of standard quantum mechanics based on the Hilbert space axiom (2.3). In Section 3 we explained that it cannot describe exponential decay and that it cannot describe resonance scattering. In Section 4 we introduced some of the Arrows of Time and discussed the quantum mechanical arrow of time in detail. We explained that time asymmetry for quantum systems is a manifestation of causality, which standard quantum mechanics based on (2.3) cannot possess. In this section we describe the steps to construct a causal theory that unifies resonance and decay phenomena. The change will affect only the dynamical evolution, which in standard quantum theory is given by the Hilbert space axiom. Most of the fundamental axioms of quantum theory will be retained. For Category I applications nothing will change. The conjecture and construction of causal quantum theory is done in two steps. The first step in this direction was already taken by most physicists when they started using the Dirac kets. The second step will demonstrate that the Gamow vectors (3.9) are the same kind of mathematical entities as the Dirac kets, except that they live in different spaces. We will show that there are many definitions of generalized vectors other than Dirac kets, including those corresponding to the Lippmann-Schwinger equation and those associated with regular and higher order resonance poles. 
Step 1: Using vectors that are not in the Hilbert space, similar to the Gamow vectors (3.9), is not without precedent. In the Hilbert space there are no Dirac kets $|E\rangle$ with the property

$$
H|E\rangle=E|E\rangle, \quad \text { for } 0=E_{0} \leq E<\infty .
$$

These Dirac kets had to be given a mathematical meaning as continuous antilinear functionals on a dense subspace $\Phi \subset \mathcal{H}$

$$
\begin{aligned}
\langle H \psi \mid E\rangle \equiv & \left\langle\psi\left|H^{\times}\right| E\right\rangle=E\langle\psi \mid E\rangle \\
& \text { for all } \psi \in \Phi \text { (but not all } \psi \in \mathcal{H}) .
\end{aligned}
$$

The continuous, antilinear functionals $F \in \Phi^{\times}$on the space $\Phi, F(\phi) \equiv\langle\phi \mid F\rangle$, form together with the antilinear functionals $h \in \mathcal{H}^{\times}$on $\mathcal{H}$, after identification $\mathcal{H}=\mathcal{H}^{\times}$(Fréchet-Riesz theorem), a Gel'fand triplet of linear topological spaces [2]

$$
\Phi \subset \mathcal{H}=\mathcal{H}^{\times} \subset \Phi^{\times} .
$$

The Dirac kets are $|E\rangle \in \Phi^{\times}$.

Typical operators for quantum mechanics (like the momentum and position operators $P$ and $Q$ and the operators $H$ for many Hamiltonians) cannot be represented by continuous (=bounded) operators in $\mathcal{H}$. However, spaces $\Phi$ can be constructed for quantum systems [2] in which the quantum mechanical operators are represented by an algebra of continuous operators (thus defined on the whole space $\Phi$ ). For every continuous operator $A$ on $\Phi$ one defines the conjugate operator $A^{\times}$on the dual space $\Phi^{\times}$by

$$
\langle A \psi \mid F\rangle=\left\langle\psi\left|A^{\times}\right| F\right\rangle \quad \text { for all } \psi \in \Phi \text { and } F \in \Phi^{\times} .
$$

It is an extension of the adjoint operator in Hilbert space. To the triplet of spaces (5.3) belongs the triplet of (selfadjoint) operators

$$
A \subset \bar{A}=A^{\dagger} \subset A^{\times}
$$

where $\bar{A}$ is the closure of $A$ in the completion of $\Phi$ to $\mathcal{H}$. The operator $A$ that fulfills $\bar{A}=A^{\dagger}$ is called essentially selfadjoint. The Dirac kets are then defined by (5.2), and in analogy to (5.1) can be written in an abbreviated way as

$$
H^{\times}|E\rangle=E|E\rangle, \quad|E\rangle \in \Phi^{\times} .
$$

To make this precise one must define the space $\Phi$ and therewith $\Phi^{\times}$. 
Most physicists and textbook authors are not concerned with the precise mathematical meaning of Dirac kets. Those who are [18] define these kets as antilinear, continuous functionals on the Schwartz space, choosing for $\Phi$ the abstract Schwartz space.

This means one replaces the Hilbert space axiom (2.3) by the following Schwartz space axiom

$$
\text { set of states }\left\{\phi^{+}\right\}=\text {set of observables }\left\{\psi^{-}\right\}=\Phi \subset \mathcal{H} \subset \Phi^{\times} .
$$

Then for every $\phi, \psi \in \Phi$ the Dirac basis vector expansion

$$
\psi=\sum_{j, j_{3}, \eta} \int \mathrm{d} E\left|E, j, j_{3}, \eta\right\rangle\left\langle E, j, j_{3}, \eta \mid \psi\right\rangle \quad \text { (and same for } \phi \text { ) }
$$

holds [2]. The $j, j_{3}, \eta$, etc, are additional quantum numbers assumed to be discrete. The Dirac kets $\left|E, j, j_{3}, \eta\right\rangle \equiv|E\rangle$ are generalized eigenvectors of $H$ in the sense of (5.2)

$$
\begin{array}{r}
\left\langle\phi\left|H^{\times}\right| E, j, j_{3}, \eta\right\rangle \equiv\left\langle H \phi \mid E, j, j_{3}, \eta\right\rangle=E\left\langle\phi \mid E, j, j_{3}, \eta\right\rangle \\
\text { for all } \phi, \psi \in \Phi .
\end{array}
$$

This is also written as

$$
H^{\times}\left|E, j, j_{3}, \eta\right\rangle=E\left|E, j, j_{3}, \eta\right\rangle .
$$

The coordinates of the basis vector expansion (5.8), or "scalar products", or brakets $\langle E \mid \phi\rangle=\overline{\langle\phi \mid E\rangle}=\phi(E)$, are smooth, rapidly decreasing functions of $E$, i.e., the energy wave functions $\phi(E)$ are "Schwartz functions" $\in \mathcal{S}_{\mathbb{R}_{+}}$. The energy wave functions $\phi(E)=\langle E \mid \phi\rangle$ define also a triplet of function spaces

$$
\{\phi(E)\}=\{\psi(E)\}=\mathcal{S}_{\mathbb{R}_{+}} \subset L^{2} \subset \mathcal{S}^{\times} .
$$

This triplet is algebraically and topologically equivalent to the triplet (5.7) of vector spaces

$$
\{\phi\}=\{\psi\}=\Phi \subset \mathcal{H} \subset \Phi^{\times} \ni|E\rangle
$$

where $\Phi^{\times}$is the space of antilinear, continuous Schwartz space functionals. The triplet (5.12) is called a Gel'fand Triplet or Rigged Hilbert Space, and the triplet (5.11) is called the "realization" of the abstract Gel'fand Triplet by the triplet of Schwartz functions.

There are many different Gel'fand triplets, and they are characterized by the choice of the vector space $\Phi$ or, equivalently, by the space of functions associated with $\Phi$. In (5.11), the Schwartz function space has been used to characterize 
the triplet. This means that the set of functions allowed in the basis vector expansion (5.8), $\phi(E)=\langle E \mid \phi\rangle=\left\langle E, j, j_{3}, \eta \mid \phi\right\rangle$, has been used to specify the space $\Phi$. Because the Schwartz functions are smooth, rapidly decreasing functions on the positive real energy axis, $0 \leq E<\infty$, the Dirac kets $|E\rangle \in \Phi^{\times}$can only have real, positive energy eigenvalues.

Having no vector in the Hilbert space with exponential time evolution, one could hope that there are kets $\phi^{G}=\left|E_{R}-\mathrm{i} \frac{\Gamma}{2}, j, j_{3}, \eta\right\rangle$ in some space $\tilde{\Phi}^{\times} \supset \mathcal{H}$, and that these kets fulfill (3.10) for every $\psi \in \tilde{\Phi}$. It means that, for $\phi^{G}$ to fulfill the exponential law for the decay probabilities, it needs to be a generalized eigenvector of the Hamiltonian $H^{\times}$and of the time evolution operator $U^{\times}(t)=\mathrm{e}^{-\mathrm{i} H^{\times}} t / \hbar$, with the eigenvalues $z_{R}$ and $\mathrm{e}^{-\mathrm{i} z_{R} t / \hbar}$ respectively

$$
\left\langle\psi\left|H^{\times}\right| \phi^{G}\right\rangle=z_{R}\left\langle\psi \mid \phi^{G}\right\rangle \quad \text { for all } \psi \text { of a space } \tilde{\Phi}
$$

and

$$
\left\langle\psi\left|\mathrm{e}^{-\mathrm{i} H^{\times} t / \hbar}\right| \phi^{G}\right\rangle=\mathrm{e}^{-\mathrm{i} z_{R} t / \hbar}\left\langle\psi \mid \phi^{G}\right\rangle \quad \text { for all } \psi \text { of a space } \tilde{\Phi} \text {. }
$$

Here, $z_{R}=\left(E_{R}-\mathrm{i} \frac{\hbar R}{2}\right)$. But the space $\tilde{\Phi}^{\times}$cannot be the Schwartz space dual of (5.7), because in the Schwartz space dual, $H^{\times}$has only real eigenvalues.

To define Gamow vectors as generalized eigenvectors, one must restrict the space of vectors $\psi$ further and admit in (5.8) only those functions $\left\langle\psi^{-} \mid E^{-}\right\rangle=\overline{\left\langle-E \mid \psi^{-}\right\rangle}$ that can be analytically continued into the lower complex plane. This is done by solving the functional energy eigenvalue equation

$$
\left\langle H \psi^{-} \mid E^{-}\right\rangle=\left\langle\psi^{-}\left|H^{\times}\right| E^{-}\right\rangle=E\left\langle\psi^{-} \mid E^{-}\right\rangle \quad \text { for all } \psi^{-} \in \Phi_{+}
$$

where we denote by $\Phi_{+}$the smaller subspace of $\mathcal{H}$ that we want to use as the space of observables: $\psi^{-} \in \Phi_{+} \subset \Phi \subset \mathcal{H}$. The space $\Phi_{+}$of allowed $\psi^{-}$, and equivalently the set of allowed energy wave functions $\left\langle{ }^{-} E \mid \psi^{-}\right\rangle=\overline{\left\langle\psi^{-} \mid E^{-}\right\rangle}$, are smaller than the space $\Phi$ in (5.7) and the set $\{\psi(E)\}$ of (5.11), respectively. Consequently the space $\Phi_{+}^{\times}$is larger than the space $\Phi^{\times}$and can have energy eigenkets with complex energy values.

Complex energy eigenvalues are suggested by the analytic S-matrix and by the Lippmann-Schwinger equation. In the theory of scattering and decay, one arrived heuristically at a pair of time asymmetric boundary conditions by choosing in- and out-plane wave "states" $\left|E^{+}\right\rangle$and $\left|E^{-}\right\rangle$, which are solutions of the LippmannSchwinger equation and are given by

$$
\left|E^{ \pm}\right\rangle=|E \pm \mathrm{i} \epsilon\rangle=|E\rangle+\frac{1}{E-H \pm \mathrm{i} \epsilon} V|E\rangle=\Omega^{ \pm}|E\rangle, \quad \epsilon \rightarrow+0
$$


Here $(H-V)|E\rangle=E|E\rangle$, and $V$ represents the scattering potential.

Because of the $\pm \mathrm{i} \epsilon$ in the Lippmann-Schwinger kets, the energy wave functions

$$
\begin{aligned}
& \psi^{-}(E)=\left\langle{ }^{-} E, j, j_{3}, \eta \mid \psi^{-}\right\rangle=\overline{\left\langle\psi^{-} \mid E, j, j_{3}, \eta^{-}\right\rangle}=\left\langle{ }^{-} E \mid \psi^{-}\right\rangle \\
& \phi^{+}(E)=\left\langle{ }^{+} E, j, j_{3}, \eta \mid \phi^{+}\right\rangle=\overline{\left\langle\phi^{+} \mid E, j, j_{3}, \eta^{+}\right\rangle}=\left\langle{ }^{+} E \mid \phi^{+}\right\rangle
\end{aligned}
$$

are respectively the boundary values of analytic functions in the upper or lower complex energy semi-plane (for complex energy $z=\overline{E+\mathrm{i} \epsilon}=E-\mathrm{i} \epsilon$ immediately below the real axis, on the 2 nd sheet of the S-matrix).

The Lippmann-Schwinger equation introduces two kinds of wave functions: $\phi^{+}(E)$ for in-states $\phi^{+} \longleftarrow \phi^{\text {in }}$ and $\psi^{-}(E)$ for out-states $\psi^{-} \longrightarrow \psi^{\text {out }}$. The in-states are the controlled in-states, defined by a preparation apparatus. In the case of the out-state vectors, it is often unclear whether an uncontrolled out-state vector, $\phi^{\text {out}}$, is meant, or whether a controlled out-state vector, $\psi^{\text {out }}$, is meant [14]. The uncontrolled out-state, $\phi^{\text {out }}$, is the result of the preparation and the scattering dynamics, whereas the controlled out-state vector, $\psi^{\text {out }}$, is defined entirely by the registration apparatus, which is built to detect specific properties of the scattering products. We shall use as $\psi^{-}$only the controlled out-state vectors. The matrix element $\left(\psi^{-}, \phi^{+}\right)$represents, then, the Born probability amplitude to register the properties $\left|\psi^{-}\right\rangle\left\langle\psi^{-}\right|$in the state described by the in-state vector $\phi^{+}$; these Born probabilities are the measured quantities.

In analogy to the Dirac expansion (5.8) the $\left|E^{ \pm}\right\rangle$are taken as basis systems for the Dirac basis vector expansion of the out-observables, $\psi^{-} \in \Phi_{+}$, and the in-states, $\phi^{+} \in \Phi_{-}$, respectively

$$
\begin{aligned}
& \Phi_{+} \ni \psi^{-}=\sum_{j, j_{3}, \eta} \int_{0}^{\infty} \mathrm{d} E\left|E, j, j_{3}, \eta^{-}\right\rangle\left\langle{ }^{-} E, j, j_{3}, \eta \mid \psi^{-}\right\rangle \\
& \Phi_{-} \ni \phi^{+}=\sum_{j, j_{3}, \eta} \int_{0}^{\infty} \mathrm{d} E\left|E, j, j_{3}, \eta^{+}\right\rangle\left\langle{ }^{+} E, j, j_{3}, \eta \mid \phi^{+}\right\rangle .
\end{aligned}
$$

The standard texts on scattering theory usually do not contain in depth discussions of the properties of the functions (5.17) and (5.18) in the basis vector expansions (5.19) and (5.20). In the Hilbert space theory one must choose them as Lebesgue square integrable functions, but then the kets $\left|E, j, j_{3}, \eta^{\mp}\right\rangle$ make no sense.

Conventionally, one also does not distinguish between the sets of in-states $\left\{\phi^{+}\right\}$ and of out-observables $\left\{\psi^{-}\right\}$, but instead equates them. Then one perhaps thinks that these two sets are both Schwartz functions: $\left\{\psi^{-}(E)\right\}=\left\{\phi^{+}(E)\right\}=\mathcal{S}_{\mathbb{R}_{+}}$. 
But the physical meaning of the two spaces $\Phi_{+}$and $\Phi_{-}$differs. The one represents detected observables, and the other represents prepared states. Therefore, their mathematical meanings need to differ as well.

Furthermore, the $\pm \mathrm{i} \epsilon$ in (5.16) suggests that the energy wave functions of the prepared state, $\left\langle^{+} E \mid \phi^{+}\right\rangle=\phi^{+}(E)$, are Schwartz functions that can be analytically continued into the lower complex energy semi-plane, and that the energy wave functions $\left\langle^{-} E \mid \psi^{-}\right\rangle=\psi^{-}(E)$ are functions that can be analytically continued into the upper complex energy semi-plane (so that the product $\left\langle\psi^{-} \mid E^{-}\right\rangle\left\langle{ }^{+} E \mid \phi^{+}\right\rangle$ can be continued into the lower complex energy plane).

Guided by the Lippmann-Schwinger equations (5.16), we take a second step away from the Hilbert space axiom (2.3).

Step 2: We distinguish mathematically between prepared states $\phi^{+}$and detected observables $\psi^{-}$. This we do by postulating that the energy wave functions $\left\langle^{+} E \mid \phi^{+}\right\rangle$ and $\left\langle^{-} E \mid \psi^{-}\right\rangle$in (5.19) and (5.20) are not only Schwartz functions on the positive real axis, but also that they can be extended to analytic functions in $\mathbb{C}_{-}$and $\mathbb{C}_{+}$, respectively. Here $\mathbb{C}_{\mp}$ are the lower and upper complex energy semiplanes of the S-matrix, which are reached by burrowing through the cut of the Riemann surface along the positive energy axis, $0 \leq E<\infty$, into the second Riemann sheet of the $\mathrm{S}$-matrix (where the resonance poles are located).

This means the energy wave functions (5.17), (5.18) in the basis vector expansions (5.19), (5.20) are not only Schwartz functions on the real energy axis, but they can also be analytically continued through the cut and into the semiplanes $\mathbb{C}_{-}$and $\mathbb{C}_{+}$, respectively. Therefore $\left\langle\psi^{-} \mid E^{-}\right\rangle\left\langle{ }^{+} E \mid \phi^{+}\right\rangle$is analytic on the semiplane $\mathbb{C}_{-}$(second sheet of the $\mathrm{S}$-matrix). This is a restriction on the energy wave functions, and it will allow analytic continuation of the S-matrix element $\left(\psi^{-} \mid \phi^{+}\right)=\left(\psi^{\text {out }}|\mathrm{S}| \phi^{\text {in }}\right)$.

This analyticity property of the wave function we generalize and idealize to the new hypothesis [1]:

$$
\begin{aligned}
& \phi^{+}(E)=\left\langle{ }^{+} E \mid \phi^{+}\right\rangle \quad \text { are Schwartz functions that can be analytically } \\
& \text { continued into the lower complex plane } \\
& \left\langle{ }^{+} E \mid \phi^{+}\right\rangle \rightarrow\left\langle{ }^{+} z \mid \phi^{+}\right\rangle \text {, } \\
& \text { precisely smooth Hardy functions on } \mathbb{C}_{-} \\
& \psi^{-}(E)=\left\langle^{-} E \mid \psi^{-}\right\rangle \quad \text { are Schwartz functions that can be analytically } \\
& \text { continued into the upper complex plane } \\
& \left\langle^{-} E \mid \psi^{-}\right\rangle \rightarrow\left\langle^{-} z \mid \psi^{-}\right\rangle \text {, } \\
& \text { precisely smooth Hardy functions on } \mathbb{C}_{+} \text {. }
\end{aligned}
$$


To conjecture (5.21) and (5.22) for $\mathbb{C}_{\mp}$ from the analyticity property suggested by the $\pm \mathrm{i} \epsilon$ in the Lippmann-Schwinger equation (5.16) (for an $\epsilon$-strip above and below the positive real energy axis) would be too farfetched. One needs additional hints from physical conditions to discover the properties (5.21), (5.22) for the wave functions. These additional conditions were conjectured from the idea (i) that exponentially decaying states fulfilling (3.3) exist, and (ii) that resonances with a Breit-Wigner energy distribution, $a_{j}^{B W}(E)$ in (3.4), have an independent significance described by a ket $\left|z_{R}^{-}\right\rangle=\left|z_{R}, j, j_{3}, \eta^{-}\right\rangle$with complex energy eigenvalue $z_{R}=E_{R}-\mathrm{i} \frac{\Gamma}{2}$.

Then one can associate with the pole of the scattering amplitude (3.4) (or the pole of the $j$-th partial S-matrix element $S_{j}^{\eta}(E)=2 \mathrm{i} a_{j}^{\eta}(E)$ for inelastic channels $\eta$ and $S_{j}(E)=1+2 \mathrm{i} a_{j}^{\eta}(E)$ for the elastic channel $\eta$ ) at complex energy $z_{R}=E_{R}-\mathrm{i} \frac{\Gamma}{2}$, a vector $\phi_{j}^{G}$ with a Breit-Wigner energy distribution

$$
\begin{aligned}
a_{j}^{B W_{i}} & =\frac{r_{\eta}}{E-z_{R_{i}}} \Longleftrightarrow \phi_{j}^{G}=\left|z_{R_{i}}, j, j_{3}, \eta^{-}\right\rangle \sqrt{2 \pi \Gamma_{i}} \\
& =\int_{-\infty}^{+\infty} \mathrm{d} E\left|E, j, j_{3}, \eta^{-}\right\rangle \frac{\mathrm{i} \sqrt{\frac{\Gamma_{i}}{2 \pi}}}{E-z_{R_{i}}} .
\end{aligned}
$$

If there are more poles in the same partial amplitude $a_{j}(z)$, this can be done for every pole $z_{R_{i}}=E_{R_{i}}-\mathrm{i} \Gamma_{i} / 2$. The vector $\left|z_{R_{i}}, j, j_{3}, \eta^{-}\right\rangle$can be shown to be an energy eigenket with a discrete, complex eigenvalue (as Gamow wanted)

$$
H^{\times}\left|E_{R_{i}}-\mathrm{i} \Gamma_{i} / 2^{-}\right\rangle=\left(E_{R_{i}}-\mathrm{i} \Gamma_{i} / 2\right)\left|E_{R_{i}}-\mathrm{i} \Gamma_{i} / 2^{-}\right\rangle, \quad\left|E_{R_{i}}-\mathrm{i} \Gamma_{i} / 2^{-}\right\rangle \in \Phi_{+}^{\times} .
$$

One calculates the probability amplitude to find an observable $\psi_{\eta}^{-}(t)$ in this generalized state $\left|E_{R}-\mathrm{i} \Gamma / 2^{-}\right\rangle$and obtains, under certain conditions on the energy wave functions $\left\langle\psi_{\eta}^{-} \mid E, j, j_{3}, \eta^{-}\right\rangle=\left\langle\psi^{-} \mid E^{-}\right\rangle$, that [1]

$$
\begin{aligned}
\left\langle\psi_{\eta}^{-}(t) \mid \phi^{G}\right\rangle & \sim\left\langle\mathrm{e}^{\mathrm{i} H t / \hbar} \psi_{\eta}^{-} \mid E_{R}-\mathrm{i} \Gamma / 2^{-}\right\rangle \\
& =\left\langle\psi_{\eta}^{-}\left|\mathrm{e}^{-\mathrm{i} H^{\times} t / \hbar}\right| E_{R}-\mathrm{i} \Gamma / 2^{-}\right\rangle \\
& =\mathrm{e}^{-\mathrm{i} E_{R} t / \hbar} \mathrm{e}^{-\frac{\Gamma t}{2 \hbar}}\left\langle\psi_{\eta}^{-} \mid E_{R}-\mathrm{i} \Gamma / 2^{-}\right\rangle \quad \text { for } t \geq 0 .
\end{aligned}
$$

From this the exponential decay law

$$
\mathcal{P}_{\phi^{G}}\left(\psi_{\eta}(t)\right)=\left|\left\langle\psi_{\eta}^{-}(t) \mid \phi^{G}\right\rangle\right|^{2}=\mathrm{e}^{-\Gamma t / \hbar}\left|\left\langle\psi_{\eta}^{-} \mid \phi^{G}\right\rangle\right|^{2}, \quad t \geq 0
$$

follows. 
The conditions for the above derivations require the properties of Hardy functions for $\left\langle^{-} E \mid \psi^{-}\right\rangle$. The time asymmetry $t \geq 0$ in (5.26) follows because $\left\langle\mathrm{e}^{\mathrm{i} H t / \hbar} \psi^{-} \mid E^{-}\right\rangle$ $=\left\langle\psi^{-}\left|\mathrm{e}^{-\mathrm{i} H^{\times} t / \hbar}\right| E^{-}\right\rangle=\left\langle\psi^{-} \mid E^{-}\right\rangle \mathrm{e}^{-\mathrm{i} E t / \hbar}$ is a Hardy function if and only if $t \geq 0$. The general time asymmetry (4.9)-(4.13) is a consequence of the PaleyWiener theorem for Hardy spaces, in the same way (2.4) and (2.5) are consequences of the Stone-von Neumann theorem for Hilbert spaces.

\section{Summary}

There is a new axiom for quantum theory. It is the Hardy space axiom

Set of prepared (in-) states de$\left\{\phi^{+}\right\}=\Phi_{-} \subset \mathcal{H} \subset \Phi_{-}^{\times}$ fined by the preparation apparatus (accelerator)

Set of (out-) observables defined

$$
\left\{\psi^{-}\right\}=\Phi_{+} \subset \mathcal{H} \subset \Phi_{+}^{\times}
$$

by the registration apparatus (detector).

The spaces $\Phi_{-}$and $\Phi_{+}$are given by the sets of vectors in (5.19) and (5.20), $\left\{\phi^{+}\right\}$and $\left\{\psi^{-}\right\}$, which are equivalent to the spaces of energy wave functions $\left\{\left\langle^{+} E \mid \phi^{+}\right\rangle\right\}=\left.\left(H_{-}^{2} \cap \mathcal{S}\right)\right|_{\mathbb{R}_{+}}$and $\left\{\left\langle^{-} E \mid \psi^{-}\right\rangle\right\}=\left.\left(H_{+}^{2} \cap \mathcal{S}\right)\right|_{\mathbb{R}_{+}}$where $H_{\mp}^{2}$ are the Hardy function spaces and $\mathcal{S}$ is the Schwartz space [6].

The spaces $\Phi_{-}$and $\Phi_{+}$are both complete with respect to a topology that is stronger than the norm-completeness of $\mathcal{H}$, and the Dirac basis vector expansion (5.19) and (5.20) can be proven as the nuclear spectral theorem for the spaces $\Phi_{-}$ and $\Phi_{+}$.

The Hardy space axiom replaces the Hilbert space axiom (2.3) as the boundary condition for the solution of the dynamical equation, (2.1) or (2.2), and leads to the semigroup evolution (4.9)-(4.13). In the relativistic case it replaces the interaction incorporating Poincaré group by semigroup representations in the forward light cone, leading to a relativistic theory of resonances and decay fulfilling Einstein causality [4]. Together with the other axioms, it provides a refinement of quantum theory by distinguishing between states and observables.

The new axiom incorporates the Category II problems and provides a mathematically consistent theory unifying resonance and decay. It also overcomes the problems associated with causality. 


\section{Acknowledgment}

This paper is based on a plenary lecture and discussions at the Workshop on Geometric Methods in Physics and Time Asymmetric Quantum Theory. We would like to thank A. Odzijewicz, P. Kielanowski and co-organizers for their hospitality. The work is part of a collaboration sponsored by US National Science Foundation Award No. OISE-0421936. The authors also acknowledge the support of the Center for Complex Quantum Systems.

\section{References}

[1] Bohm A., Resonance Poles and Gamow Vectors in the Rigged Hilbert Space Formulation of Quantum Mechanics, J. Math. Phys. 22 (1981) 2813-2823; Gamow State Vectors as Functionals Over Subspaces of the Nuclear Space, Lett. Math. Phys. 3 (1978) 455-461.

[2] Bohm A. and Gadella M., Dirac Kets, Gamow Vectors and Gel'fand Triplets, Lecture Notes in Physics, vol. 348, Springer, Berlin, 1989.

[3] Bohm A., Quantum Mechanics: Foundations and Applications, 3rd Edition, Springer, N.Y., 2001.

[4] Bohm A., Kaldass H. and Wickramasekara S., Time Asymmetric Quantum Theory - II. Relativistic Resonances from S-matrix Poles, Fortschr. Phys. 51 (2003) 569-603; Time Asymmetric Quantum Theory - III. Decaying States and the Causal Poincaré Semigroup, 51 (2003) 604-634.

[5] Feynman R., Space-Time Approach to Non-Relativistic Quantum Mechanics, Rev. Mod. Phys. 20 (1948) 367-387.

[6] Gadella M., A Rigged Hilbert Space of Hardy-class Functions: Applications to Resonances, J. Math. Phys. 24 (1983) 1462-1469.

[7] Gamow G., Zur Quantentheorie des Atomkernes, Z. Phys. 51 (1928) 204212.

[8] Gell-Mann M. and Hartle J., in Physical Origins of Time Asymmetry, J. Halliwell et al. (Eds.) Cambridge, 1994; Gell-Mann M. and Hartle J., UCSBTH95-28, University of California at Santa Barbara, 1995; gr-qc/9509054 [and references therein]; Bohm A., Time-asymmetric Quantum Physics, Phys. Rev. A 60 (1999) 861-876.

[9] Kapur P. and Peierls R., The Dispersion Formula for Nuclear Reactions, Proc. Roy. Soc. London, Ser. A, Math. and Phys. Sci. A166 (1938) 277295; R. Peierls, Proceedings of the 1954 Glasgow Conference on Nuclear 
and Meson Physics, Bellamy E. et al. (Eds), Pergamon Press, New York, 1955; Garcia-Calderon G. and Peierls R., Resonant States and Their Uses, Nuclear Physics A 265 (1976) 443-460; Hernandez E. and Mondragon A., Resonant States in Momentum Representation, Phys. Rev. C 29 (1984) 722738; Mondragon A. and Hernandez E., Resonances and Gamow States in Non-Local Potentials, Ann. der Physik 503 (1991) 503-517.

[10] Khalfin L., Contribution to the Decay Theory of Quasi-stationary State, Sov. Phys. JETP 6 (1958) 1053-1063.

[11] Levy M., On the Description of Unstable States in Quantum Field Theory, Nuovo Cim. 13 (1959) 115-143.

[12] Ludwig G., Foundations of Quantum Mechanics, vol. I, Springer, Berlin, 1983 and vol. II, 1985; An Axiomatic Basis of Quantum Mechanics, vol. I Springer, Berlin, 1983 and vol. II, 1987; Kraus K., State, Effects and Operations, Springer Lecture Notes in Physics, vol. 190, Springer, Berlin, 1983.

[13] [a] Merzbacher E., Quantum Mechanics, Ch. 18, Wiley, New York, 1970; [b] Cohen-Tannoudji C., Diu B. and Laloe F., Quantum Mechanics, vol. II, p. 1345, pp. 1353-54 Wiley, New York, 1977; [c] Lee T., Particle Physics and Introduction to Field Theory, Ch. 13, Harwood Academic, New York, 1981.

[14] Newton R., Scattering Theory of Waves and Particles, 2nd Ed., in particular Ch. 7, McGraw-Hill, New York, 1966.

[15] Norman E., Gazes S., Crane S., and Bennett D., Tests of the Exponential Decay Law at Short and Long Times, Phys. Rev. Lett. 60 (1988) 2246-249.

[16] Peierls R., Surprises in Theoretical Physics, Princeton University Press, Princeton, 1979.

[17] Stone M., On One-Parameter Unitary Groups in Hilbert Space, Ann. Math. 33 (1932) 643-648; von Neumann J., Über Einen Satz von Herrn M. H. Stone, Ann. Math. 33 (1932) 567-573.

[18] Streater R. and Wightman A., PCT, Spin and Statistics and All That, Ch. 2, Princeton University Press, Princeton, 1980; Haag R., Local Quantum Physics, Ch. II, Springer, Berlin, 1992.

[19] von Neumann J., Mathematical Foundations of Quantum Mechanics, German Edition, Springer Berlin, 1931, English Edition, Princeton University Press, Princeton, 1995.

[20] Weisskopf V. and Wigner E., Berechnung der natürlichen Linienbreite auf Grund der Diracschen Lichttheorie, Z. Physik 63 (1930) 54-73; Über die 
natürliche Linienbreite in der Strahlung des harmonischen Oszillators, 65 (1930) 18-29.

[21] Goldberger M. and Watson K., Collision Theory, Ch. 8, John Wiley, N.Y., 1967.

Arno Bohm

CCQS, Department of Physics

University of Texas at Austin

Austin, TX 78712

USA

E-mail address: bohmephysics.utexas.edu

Peter Bryant

CCQS, Department of Physics

University of Texas at Austin

Austin, TX 78712

USA

E-mail address: pbryant @physics . utexas .edu 